\title{
Counseling new mothers on weight loss: a review of the most effective strategies \& suggestions for counseling
}

\begin{abstract}
Obesity is on the rise and is growing at a rapid rate. The nation must address strategies to slow down. One important trigger for obesity among women is pregnancy weight that is not lost within 6months postpartum (PP). Weight retention during the PP period is one of the greatest predictors of long-term weight gain and thus increases a woman's obesity risk. It is critical that women lose weight during the PP period to decrease obesity risk and improve health outcomes. The aim and purpose of this paper is to review the literature on the barriers new mothers face when attempting to achieve weight loss and the best counseling techniques professionals can use to help guide their success. Particular groups of women are at greater risk of PP weight gain and retention. New mothers experience many lifestyle changes, increasing their risk of stress and weight retention. They also face many barriers to weight loss including time, prioritizing, financial constraints, lack of sleep, depression, and lack of support. For counseling to be Obesity is on the rise and is growing at a rapid rate. The nation must address strategies to slow down. One important trigger for obesity among women is pregnancy weight that is not lost within 6months postpartum (PP). Weight retention during the PP period is one of the greatest predictors of long-term weight gain and thus increases a woman's obesity risk. It is critical that women lose weight during the PP period to decrease obesity risk and improve health outcomes. The aim and purpose of this paper is to review the literature on the barriers new mothers face when attempting to achieve weight loss and the best counseling techniques professionals can use to help guide their success. Particular groups of women are at greater risk of PP weight gain and retention. New mothers experience many lifestyle changes, increasing their risk of stress and weight retention. They also face many barriers to weight loss including time, prioritizing, financial constraints, lack of sleep, depression, and lack of support. For counseling to be effective for new mothers all of the discussed barriers women face during that postpartum period need to be addressed. The counseling intervention must be convenient, time sensitive, affordable, and provide support. Suggested counseling strategies include home-based interventions that are time sensitive and flexible. Also, effective counseling interventions should be affordable, creating relevant and realistic goals, and provide support. Future research is needed on the most effective technology-based and home-based interventions.
\end{abstract}

Keywords: PP, postpartum; GDM, gestational diabetes mellitus; WIC, women, infants and children
Volume 4 Issue I - 2016

\author{
Katherine Turner \\ Philadelphia Freedom Valley YMCA, USA
}

Correspondence: Katherine Turner, Philadelphia Freedom Valley YMCA, 7813 Germantown Ave Philadelphia, PA 19118 USA, Tel 6103064679, Email kkturner22@gmail.com

Received: March 20, 2015 | Published: January 04, 2016

\section{Background}

Obesity is a growing epidemic in the U.S and it doesn't seem to be slowing down. In 2009 alone, about 2.4million more U.S adults were obese than in 2007 and the most recent census concluded that more than one-third (78.6million) of U.S adults are obese. In every state more than $15 \%$, the national goal, of adults are obese and in 9 states over $30 \%$ of adults are obese. Some obesity-related conditions include cardiovascular disease, stroke, type 2 diabetes, and certain types of cancer; these are some of leading causes of death in the U.S. Not only is there a cost to one's health associated with obesity, but there is also a physical cost as well. In 2008, the medical costs for people who were obese were $\$ 1,429$ higher per person than those of normal weight, placing a huge financial burden on our medical care system.

Obesity is prevalent in some groups more than others. NonHispanic blacks have the highest rates of obesity (47.8\%) followed by Hispanics $(42.5 \%)$, non-Hispanic whites $(32.6 \%)$, and non-
Hispanic Asians (10.8\%). It is also higher amount middle age adults, 40-59years old than among younger adults 20-39years old or adults over 60years old. In terms of socioeconomic status, no relationship is found between obesity and education among men. However, among women, there is a growing relationship between those with college degrees and a decreased risk for obesity compared with less educated women. Higher income women are also at decreased risk for obesity compared to low-income women.

People's social and physical environments can contribute greatly to their weight status. Some Americans do not have access to stores that provide healthy, affordable food, such as fresh fruits and vegetables. However, they may have increased access to snack shops, fast-food restaurants, and unhealthy vending machines. Many urban environments lack safe community parks or sidewalks, making it difficult to increase physical activity. Behavior also plays an important role in determining one's health and weight status. As a country eating behaviors have changed. The U.S has become a nation obsessed with 
convenience foods, neglecting their detrimental effects. Americans eat out at restaurants more than preparing meals at home. Foods high in sugar, fat, and salt are highly advertised and are often easier and cheaper their healthier counterpart. Our unfavorable evolving environments and behaviors have been identified as the greatest areas for prevention and treatment actions. It is important to note that providing one with knowledge does not always enlist lifestyle changes. Rather focusing on behavior change and environmental/ social change has been shown to be more effective.

Obesity is growing fast and the nation needs to identify strategies to slow it down. Two important triggers for obesity among women are excessive weight gain during pregnancy and pregnancy weight that is not lost within 6months postpartum (PP). ${ }^{1}$ Postpartum weight retention is defined as the difference between postpartum and prepregnancy weight. Weight retention is one of the most significant predictors of long-term weight gain. ${ }^{2}$ It is critical that women lose weight during their firstyear PP in order to prevent excess weight retention and gain. Therefore, weight loss interventions among PP women are an important component in reducing the prevalence of obesity and improving health outcomes. The aim and purpose of this paper is to review the literature on the barriers new mothers face when attempting to achieve weight loss and the best counseling techniques professionals can use to help guide their success.

\section{Characteristics of postpartum women}

Postpartum women are more likely to gain and retain weight, related to both pregnancy itself and due to a slowing metabolism during childbearing years. On average women retain about $3 \mathrm{~kg}$ (6.6lbs) per pregnancy over the course of their life, increasing their risk for obesity with each pregnancy. ${ }^{3}$ In a study reviewed by Ko Y et al., ${ }^{2}$ it was shown that women who were able to lose the weight they gained during pregnancy were only $2.4 \mathrm{~kg}$ (5.3lbs) heavier at 10 years follow-up. However, the women who retained their weight by 6 months PP were on average $8.3 \mathrm{~kg}$ (18.31bs) heavier at 10years follow-up. It was also found in another study that women who retained at least $4.5 \mathrm{~kg}(9.91 \mathrm{bs})$ had a $14-25 \%$ chance of developing obesity. ${ }^{2}$ Unfortunately $60 \%$ of women cannot return to their pre-pregnancy weight by 6 months PP, putting them at greater risk for developing obesity and obesity-related conditions. Certain groups of women are at greater risk for being within this $60 \%$ than others. Low socioeconomic status \& minority women are at greater risk of PP weight gain and retention. This could be related to the fact that non-Hispanic black women and Hispanics have the highest rates of obesity, $41.9 \%$ and $30.7 \%$ respectively. Single, low-income women less than $20 y e a r s$ old or greater than 40years old also retain significantly more weight in the postpartum period. ${ }^{1}$ This outcome can be attributed to behavioral and environmental factors such as lack of support, financial constraints, unavailability of health promoting resources, and immaturity.

Gestational Diabetes Mellitus (GDM) affects up to $14 \%$ of pregnancies in the U.S. Women with GDM are typically at an even greater risk. A diagnosis of GDM doubles the risk of developing type 2 diabetes within 4 months PP. ${ }^{4}$ It is imperative that women with GDM lose most of their PP weight even before 6months PP in order to prevent the onset of type 2 diabetes. Women with GDM also have a $41 \%$ risk of developing GDM again in later pregnancies and therefore must lose their PP weight with each new pregnancy. ${ }^{4}$

Postpartum women are experiencing a great deal of changes during this time. They are adjusting to not only lifestyle and physical changes, but mental changes as well. A new mother's life is centered around her infant, which lead to increased stress. This is can be especially true for mothers when it is their first-born child. This increased stress risk, in PP women, can play a large role in their weight loss success.

\section{Barriers to weight loss}

There are many barriers PP women face when trying to make healthy lifestyle changes including time, prioritizing, financial constraints, lack of sleep, depression, and lack of support. Time is one of the most common barriers among PP women. In majority of the studies reviewed many participants could not find the time to fulfill the study's requirements due to time constraints. One participant said that an exercise and behavior change program was just another thing to add in to her already packed schedule. ${ }^{5}$ Another participant had difficulty trying to find time to eat healthy during the day and felt that anything that took her away from her children was just another time commitment, similar to the pervious participant. ${ }^{5}$ In the same study, participants expressed the need for more time to plan out healthy meals and more time to go to the grocery store without distractions. Many women who returned to work during the PP period found it even more difficult to find the time to make healthy lifestyle changes. One women said that when she went back to working full-time she ate whatever her children were eating because she did not have time to prepare separate meals. ${ }^{6}$ Another participant in the same study said that she felt guilty for being away from her children, while she was working so she didn't make time to exercise. Also, finding convenient and affordable childcare to meet the mother's needs can be a struggle. Nicklas et al., ${ }^{6}$ found that lack of time and lack of childcare were the two most important barriers to healthy lifestyle activities in PP women with prior GDM. Along with time, prioritizing one's time can be a barrier to weight loss. Many new mothers prioritize their infant's and children's needs over their own. In a study conducted by CarterEdwards et al., ${ }^{5}$ all participants expressed that at the current point in their lives their personal health was not a top priority. One participant also said that her children's welfare should take precedence over her taking care of her own health. There are many other barriers associated with time. Montgomery et al., ${ }^{7}$ identified six themes related to time including food choices, school, career, exercise, multiple children, and infant care responsibilities. It is evident that time and perceived priorities can be one of the largest barriers for PP mothers when trying to make healthy changes.

Financial constraints can be a barrier for some women. Nicklas et al., ${ }^{6}$ found that when it came to participants trying to eat healthier, many participants reported that the cost of shopping is too high and that the salad bar costs more than fast food. When it came to exercise, participants reported that gym memberships are just too expensive. Similarly, in a study conducted by Setse et al., ${ }^{8}$ the most common reported barrier to weight loss and participation in weight loss programs following pregnancy was cost.

Postpartum depression and lack of sleep are other aspects that can pose as barriers to PP women when trying to lose weight. It was found that women who reported 5hours of sleep or less in a 24-hour period at 6 months PP were 2.3 times more likely to retain a minimum of $5 \mathrm{~kg}$ (11lbs) body weight at lyear PP, regardless of sociodemographics, pre-pregnancy weight, gestational weight gain, and postpartum behaviors. ${ }^{1}$ Nicklas et al., ${ }^{6}$ also found that many participants reported a barrier to getting enough exercise was constant fatigue and feeling tired, not only physically, but mentally as well. Postpartum depression affects $21.9 \%$ of women during the first year PP. ${ }^{9}$ Women reported 
that their PP depression caused them to uncontrollably eat at times. ${ }^{8}$ In a study conducted by Montgomery et al., ${ }^{7}$ some participants reported that their PP depression led them to want to sleep constantly and another stated that her anxiety was so high she "worried the weight off," but go to a point where she was so fatigued worrying that she didn't have time to eat.

Along with PP depression and lack of sleep, lack of support from family members, friends, co-workers, and health providers plays a large role in weight retention. Women who did not have significant support systems were at a greater risk of long-term weight gain following delivery. ${ }^{1}$ Montgomery et al., ${ }^{7}$ identified common themes from their participant interviews including: lack of support, support outside the family, self-support, family (other than husband) support, support of health care providers, and support of husband. Participants found that when they did receive support from their social or family circle it instilled more self-confidence to lose weight. According to Montgomery et al., ${ }^{7}$ a participant found it helpful when other people cooked meals so she didn't have to and another mother found motivation to exercise through starting a friendly competition with another PP mother. Support from husbands and other family members came in the form of compliments and helping with diet exercise, and babysitting. In another study, conducted by Carter-Edwards et al., ${ }^{5}$ many of the participants lacked social and family support. One participant felt that she did not receive strong support from her husband and felt uncomfortable asking him to care for multiple children at one time. The same participant also felt guilty when she missed work due to a child's illness, but felt support when her coworkers were willing to switch shifts with her. All of the study's participant's support networks were either important in motivating them to engage in healthy lifestyle behaviors, or were perceived as barriers.

\section{Counseling needs}

For counseling to be effective for new mothers all of the discussed barriers women face during that postpartum period need to be addressed. The counseling intervention must be convenient, time sensitive, affordable, and provide support. The location must be convenient for their family's day-to-day life. Participants expressed that in order for them to be successful in the program it would have to be located close to home. They also noted that child-care services would need to be offered during any weight loss intervention, so they would be able to go and not need to get a babysitter. ${ }^{8}$ Carter-Edwards et al.,$^{5}$ had similar findings where most women reported that time to drive to classes, coupled with no child care, made it difficult to participate in weight loss programs. New mothers need weight loss interventions that are convenient for them to get to and are able to accommodate their children. These interventions must also be timesensitive and flexible. One participant stated, "timing is key, we don't have much of it". ${ }^{6}$ Montgomery et al., ${ }^{1}$ concluded that new mothers need to balance taking care of their newborn with other family demands, while at the same time trying to return to a state of health and normalcy. This can make it very challenging for them to fit in exercise and other healthy lifestyle changes.

Along with convenience and flexibility, weight loss interventions and suggestions must also be affordable. It was a reoccurring theme among study participants that both gym memberships and healthy foods were too expensive. Weight loss suggestions made when counseling must be realistic. Suggesting a client belong to a gym when they cannot afford it is not helpful and can be discouraging to the client. Also suggesting fruits and vegetables when they are not in season and are more expensive is not the best strategy if a client is already strained financially.

The need for support also plays a large role in weight loss success. New mothers need the support of their family, friends, co-workers, and healthcare providers. According to Montgomery et al., ${ }^{7}$ support plays an essential role in women's experience with postpartum weight loss. Getting the client's support network involved in the intervention can be an effective strategy for weight loss. In an effort to seek support from family members, participants wanted their spouse or partner included in the intervention so they could support their lifestyle changes. Some participants in the same study wanted their entire family/household included in the intervention so everyone could be on board. Along with wanting their family involved, some of these women also expressed that they felt judged by clinicians about their lifestyle and choices. ${ }^{6}$ Clinicians are a part of a new mother's support network and need to be mindful of their approach to counseling this population. As counselors, it may not be possible to change the support that clients receive from their friends, family members, coworkers, etc. However, counselors can be the client's main support during the postpartum period.

\section{Suggestions \& strategies for counseling}

\section{Counseling setting}

In terms of location, any nutrition intervention should be convenient for the client to physically get to. Ostbye et al., ${ }^{10}$ concluded that community-based interventions delivered outside the home are unlikely to impact postpartum weight loss, and more accessible programs delivered in the home via telephone, mail, or internet/e-mail may be easier for and therefore more successful. One of the greatest barriers or limitations for a majority of the studies reviewed was lack of full participation, due to convenience and time constraints. Nicklas et al., ${ }^{6}$ had similar conclusions, stating that a lifestyle intervention program that is primarily internet-based that includes a lifestyle coach may be feasible and acceptable for postpartum women with a history of GDM. These suggested home-based interventions include a counselor meeting the client at their home or interventions using the telephone, mail, or internet/e-mail. Telephone and email can also be used for client accountability. Conducting regular "check-ins" with a client can provide them with continuity of care and aid in their weight loss. Home and technology based interventions are great solutions to the common barrier of convenience. Another aspect of convenience that must be addressed is childcare. If an intervention includes counseling sessions away from the home or exercise interventions at a gym, it is important that childcare is provided.

\section{Time sensitivity}

Counselors must be flexible in their timing of interventions. The counselor must accommodate the client and offer various convenient times to ensure full participation. Informing the client that their appointment is at their convenience may also offer an area of support for them. Because new mothers are strained for time, it is important to make eating healthy and exercising fun for them not just one more thing they have to add into their already packed schedule. ${ }^{5}$ In order to do this the counselor will have to get to know the client and find out what they enjoy and tie that into their healthy lifestyle changes. The counselor should also focus on meals and snacks that take little preparation and have easy clean up, and quick, effective exercise workouts. 


\section{Realistic \& relevant goal setting}

Counselors need to make sure goals are relevant and realistic when goal setting with the client. The client's available resources need to be taken into account, for example whether they have fresh fruits and vegetables readily available in their neighborhood, identifying where they grocery shop and what the store has to offer, and what can the client can afford. Healthy eating and exercise goals must be time-sensitive. Meal suggestions that require time-consuming meal preparation will not be suitable for this population; everything must be quick and easy. Strategies for exercising, that doesn't require a gym membership, may be needed for women who cannot afford one or if their gym does not offer childcare, making it hard for them to go. The counselor must also take into account where the client lives and if have a safe environment for outdoor physical activity.

\section{Support}

Nicklas et al., ${ }^{6}$ discussed the importance of social supports being involved in a lifestyle intervention program. However, group sessions typically did not work due to timing and convenience. Providing home-based interventions that include social support (partner, family, friends, etc.) for the client would be a more effective solution. Having family cooking classes at their house can be a good way to get everyone involved and teach healthy cooking skills. Also exercise strategies that involve a social support, like walking with a friend, should be recommended. ${ }^{11}$ As stated previously, it can be challenging to improve the client's support network and then counselor may be the main support. It's important to note that if the client is struggling with barriers like depression, the first couple of sessions many not be about weight loss, but it is critical to address these barriers prior to discussing strategies for weight loss for the intervention to be effective.

\section{Resources}

Resources that may be helpful to the client include Postpartum Support International, lactation consultants and counselors, Women, Infants and Children (WIC), Local Harvest and information on their local YMCA. Postpartum Support International provides support to postpartum women suffering from perinatal mood and anxiety disorders, including postpartum depression. Lactation consultants and counselors can be great resources for new mothers having difficulty breastfeeding. WIC is a supplemental nutrition program that can take on some of the financial burden new mothers face. WIC provides vouchers for healthy foods, aiding in the selection of healthy choices, which can promote weight loss. Local Harvest lists all the farmers markets in a particular area; all one has to do is put in their zip code. Local YMCAs offer a variety of financial assistance plans to ensure that everyone can afford a YMCA membership. Some branches also offer free daily childcare, allowing mothers to drop their child off and workout. Please refer to Appendix A for more information about each of these resources.

\section{Conclusion \& future research}

An important strategy to slow down the growing rates of obesity is to target postpartum weight loss. New mothers face many barriers and challenges to weight loss and increase physical activity. Providing convenient, time-sensitive and affordable solutions can have a significant impact on their weight loss success. Also, enhancing and providing support to the client plays a key role. Future research is needed to more closely assess the effectiveness of technology-based interventions, determining which technology-based intervention is the most effective for different populations. Future research is also needed in in-home interventions, where the counselor actually goes to the client's home. Suggested interventions include, in-home pantry raids and community grocery shopping tours.

\section{Acknowledgements}

None.

\section{Conflict of interest}

The author declares no conflict of interest.

\section{References}

1. Montgomery KS, Aniello TD, Phillips JD, et al. Experiences Accompanying postpartum weight loss: benefits, successes, and wellbeing. Health Care Women Int. 2013;34(7):577-591.

2. Ko YL, Yang CL, Fang CL, et al. Community-based postpartum exercise program. J Clin Nurs. 2013;22(15-16):2122-2131.

3. Rosal MC, Lemon SC, Nguyen $\mathrm{OH}$, et al. Translation of the diabetes prevention program lifestyle intervention fro promoting postpartum weight loss among low-income women. Transl Behav Med. 2011;1(4):530-538.

4. Kaiser B, Razurel C. Determinants of postpartum physical activity, dietary habits and weight loss after gestational diabetes mellitus. Journal of Nursing Management. 2013;21(1):58-69.

5. Carter-Edwards L, Østbye T, Bastian LA, et al. Barriers to adopting a healthy lifestyle: insight from postpartum women. BMC Res Notes. 2009;2:161.

6. Nicklas JM, Zera CA, Seely EW, et al. Identifying postpartum intervention approaches to prevent type 2 diabetes in women with a history of gestational diabetes. BMC Pregnancy and Childbirth. 2011;11:23.

7. Montgomery KS, Bushee TD, Phillips JD, et al. Women's challenges with postpartum weight loss. Matern Child Health J. 2011;15(8):1176-1184.

8. Setse R, Grogan R, Cooper LA, et al. Weight loss programs for urbanbased, postpartum African-American women: perceived barriers and preferred components. Matern Child Health J. 2008;12(1):119-127.

9. Wisner KL, Sit DK, McShea MC, et al. Onset timing, thought of self-harm, and diagnoses in postpartum women with screen-positive depression findings. JAMA Psychiatry. 2013;70(5):490-498.

10. Østbye T, Peterson BL, Krause KM, et al. Predictors of postpartum weight change among overweight and obese women: results from the active mothers postpartum study. $J$ Womens Health. 2012;21(2):215-222.

11. Groth SW, David T. New mothers' views of weight and exercise. $M C N$ Am J Matern Child Nurs. 2008;33(6):364-370. 